\title{
Fetal stage melanopsin (OPN4) and GNAQ (Gaq) signaling regulates vascular development of the eye
}

Shruti Vemaraju ${ }^{1}$, Gowri Nayak${ }^{1}$, William E. Miller ${ }^{6}$, David R. Copenhagen ${ }^{5}$ and Richard A. Lang ${ }^{1-}$ $4^{*}$

${ }^{1}$ The Visual Systems Group, Abrahamson Pediatric Eye Institute, Division of Pediatric Ophthalmology, ${ }^{2}$ Division of Developmental Biology, ${ }^{3}$ Center for Chronobiology, Cincinnati Children's Hospital Medical Center, Cincinnati, OH 45229, USA

${ }^{4}$ Department of Ophthalmology, University of Cincinnati, College of Medicine, Cincinnati, $\mathrm{OH}$ 45229, USA

${ }^{5}$ Departments of Ophthalmology and Physiology, University of California, San Francisco, San Francisco, California 94158, USA.

${ }^{6}$ Department of Molecular Genetics, University of Cincinnati, College of Medicine, Cincinnati, $\mathrm{OH}$ 45229, USA

\section{Author contributions}

$\mathrm{DRC}$ and RAL provided project leadership, designed experiments and supervised experimental work. RAL, DRC and SV wrote the manuscript. WEM provided crucial reagents and advice. SV and GN performed experimentation and analysis.

\section{* Corresponding author:}

Richard A. Lang, Division of Pediatric Ophthalmology, Cincinnati Children's Hospital Medical Center, 3333 Burnet Avenue, Cincinnati, OH 45229, USA. Tel: 513-636-2700 (Office), 513-8032230 (Assistant), Fax: 513-636-4317. Email: Richard.Lang@cchmc.org 


\begin{abstract}
Maturation of sensory systems in mammals is regulated by appropriate sensory stimulation. Developmental refinement of the eye and visual system is regulated by light and visual stimulation. One compelling example is that fetal mouse pups deprived of light exhibit altered vascular development in their eyes. Previous work demonstrated that light activation of the photopigment melanopsin (Opn4), an atypical opsin expressed in intrinsically photosensitive retinal ganglion cells (ipRGCs), is crucial to normal vascular development. This suggested the unusual hypothesis that vascular development of the eye was regulated by ipRGC responses in the fetal eye by light that traveled through the body wall of the mother. Here, we test the requirement of OPN4 during fetal stages using genetic approaches. The G-protein GNAQ (Gaq) is a candidate mediator of melanopsin signaling. We show that ipRGC-specific deletion of Gnaq phenocopies both hyaloid and retinal vascular development of the Opn4 null mouse. Furthermore, GNAQ gain-of-function in Opn4-expressing cells only during late gestation was sufficient to reverse the consequences for vascular development of either dark rearing or Opn4 loss-offunction. We conclude that melanopsin-dependent signaling in the fetal mouse eye is necessary and sufficient for vascular maturation.
\end{abstract}

\title{
Introduction
}

Melanopsin is a G-coupled receptor that, like other opsins ${ }^{1-4}$, initiates a signaling cascade when it captures a photon of light. Melanopsin was discovered in the melanophores of the frog Xenopus laevis ${ }^{5}$ but is now known to be present in most vertebrates ${ }^{6}$. In adult mice, melanopsin mediates circadian entrainment ${ }^{7-10}$ and the pupillary light reflex ${ }^{9,11}$. More recently, melanopsin was shown to be essential for negative phototaxis in neonates ${ }^{12}$ and for normal vascular development of the eye ${ }^{13}$.

In mammals, melanopsin is expressed in so-called intrinsically photosensitive retinal ganglion cells (ipRGCs). The axons of ipRGCs project to several regions of the brain, such as the suprachiasmatic nucleus (SCN $)^{14-18}$ and the olivary pretectal nucleus ${ }^{11,17,19-22}$, that are consistent with the light-sensing physiological functions of melanopsin. It has been shown that melanopsin expressing cells are photo-sensitive as early as the day of birth (P1) in the mouse ${ }^{23}$. This is 9 days prior to the emergence of visual signaling from conventional photoreceptors, consistent with the acute action of melanopsin in the negative phototaxis reflex of mice at $P 5^{12}$. Several studies have demonstrated that ipRGCs express Gnaq/11 subfamily members ${ }^{24-26}$ and that melanopsin 
phototransduction is predominantly mediated by $\mathrm{Gnaq} / 11$ coupling ${ }^{24,27,28}$. Other reports suggest that melanopsin functions independent of $\mathrm{Gnaq} / 11$ both in vivo ${ }^{26}$ and in vitro ${ }^{29-31}$. The in vivo studies were performed in neonatal pups or adult animals, and the nature of melanopsin phototransduction in fetal retina remains to be explored.

Melanopsin is required for normal vascular development of the mouse eye ${ }^{13}$. Mice that are dark reared from E15 or that are Opn4 loss-of-function show promiscuous retinal angiogenesis and hyaloid vessel persistence ${ }^{13}$. A model explaining melanopsin-dependent vascular development suggests that the first step is the suppression of retinal neuron numbers ${ }^{13}$. It is proposed that in Opn4 null and dark reared mice, abnormally high numbers of retinal neurons induce a hypoxia response that results in high expression of vascular endothelial growth factor $A$, $(\text { VEGFA })^{13}$, a key regulator of developmental angiogenesis. In turn, high levels of VEGFA are proposed to cause promiscuous angiogenesis in the retina as well as to suppress regression of the hyaloid vessels ${ }^{13}$. One unusual outcome of this analysis was the proposal, based on staged dark rearing experiments, that the light response window for melanopsin-dependent vascular development occurs during gestation between $\mathrm{E} 16$ and $\mathrm{E} 18^{13}$. This further implied that melanopsin in the fetal retina would be stimulated by light that traveled through the body wall of the dam ${ }^{13}$. Measured levels of light within the visceral cavity of adult female mice were consistent with this possibility given the threshold photo-sensitivity of ipRGCs ${ }^{23,32,33}$.

In the current study we have used experimental strategies to address the role of melanopsin signaling during fetal mouse stages in regulating vascular development in the eye. We show that gain- and loss-of-function modulation of GNAQ (Gaq) in Opn4-expressing cells results in changes in vascular development. Importantly, Gnaq loss-of-function phenocopies lossof-function for Opn4 while GNAQ gain-of-function restricted to fetal Opn4-expressing cells reverses the vascular consequences of dark rearing and Opn4 loss-of-function. These data make a strong case that melanopsin function via GNAQ signaling during fetal stages is necessary and sufficient for vascular maturation in the eye. Given the OPN4 gene is expressed at gestational week 9 in human eyes ${ }^{34}$, these findings also raise the very interesting possibility that this same pathway may be conserved in the human fetus.

\section{Results}

\section{Gnaq loss-of-function in Opn4-expressing cells phenocopies Opn4 mutation}

In order to employ manipulations of G-protein signaling to investigate fetal ipRGC vascular development responses, we first needed to establish which G-protein was involved. If ipRGCs 
employ GNAQ for melanopsin phototransduction, then a deletion of Gnaq in Opn4-expressing cells in mice should reproduce the vascular development phenotype of the Opn4 null ${ }^{13}$. To test this, we combined the Opn $4^{\text {cre }}$ allele with $G n a q^{f l}$ (officially $G n a q^{\text {tm2Soff }}$ ) but also included the germline loss-of-function Gna11 allele because GNAQ and GNA11 frequently function together. To assess the consequences of deletion of GNAQ and GNA11 in Opn4-expressing cells, we generated Opn4 $4^{\text {cre }}$; Ai14, Gna11/\% Gnaq $^{f / / f l}$ mice and assessed deletion using immunofluorescence detection of GNAQ and GNA11. ipRGCs in the retina were identified by the expression of tdTomato from the Ai14 cre reporter (Fig. 1A-E). Use of a single antibody that detects both GNAQ and GNA11 on cryosections from retina revealed that control ipRGCs showed membrane immunoreactivity (Fig. 1A-E), but almost all those from mice with combined Gna11 and Gnaq mutations did not (Fig. 1F-H). A few ipRGCs retained GNAQ/11 labeling (Fig. 1G, H, red arrow) and probably represent cells that did not recombine both $G_{n a q} q^{f l}$ alleles in response to Opn4 ${ }^{\text {cre }}$. With this evidence for GNAQ/11 loss in ipRGCs, we analyzed the eyes of mutant mice for vascular anomalies.

The hyaloid vessels are fetal blood vessels that reside transiently between lens and retina. These vessels undergo a maturational regression, presumably as an adaptation to clear the visual axis for high-acuity vision. In the mouse this occurs between postnatal $(P)$ days 1 and $10^{35}$. Counts of vessel numbers at $\mathrm{P} 8$ provides a simple and reliable read-out for regression activity ${ }^{13,36}$. In P8 Gnaq $^{f / /+}$; Gna11+/- control mice, we observed hyaloid vessel numbers consistent with a normal

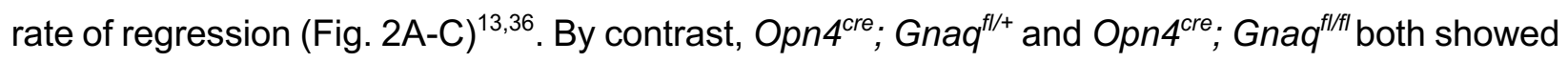
hyaloid persistence. Deleting both alleles of Gnaq produced the highest level of persistence suggesting a gene dose-dependence (Fig. 2A-C). The addition of Gna11 germ-line homozygosity to Gnaq conditional deletion in ipRGCs did not further elevate hyaloid vessel number at P8 (Fig. 2C). These data suggest GNAQ is required in Opn4-expressing cells for activity of the pathway that controls hyaloid regression. The data also suggest that GNA11 is unlikely to be a significant contributor.

As previously shown Opn4 deletion results in persistent hyaloid vessels at $\mathrm{P} 8^{13}$. For comparison purposes we also show here that Opn4 homozygote null mice show a significant elevation in the number of hyaloid vessels (Fig. 2D). The absolute numbers of hyaloid vessels in control animals shown in Fig. $2 \mathrm{C}$ and $2 \mathrm{D}$ is distinct and this very likely reflects differences in strain background. Namely, the data in Fig. $2 \mathrm{C}$ are the result of a mixed background cross and that in Fig. 2D is C57BL/6 background. Any changes in hyaloid vessel number are thus best compared as a proportion of the value from littermate controls. When we express hyaloid vessel number for both the germline Opn4 and conditional Gnaq mutants as a proportion of control (white numbers, 
Figs. 2C, D), we find that GNAQ loss-of-function produces a phenotype that is at least as severe as that for OPN4. This argues that for this vascular development pathway GNAQ activity can fully account for the signaling activity of OPN4.

During the postnatal period in mice, the retina becomes vascularized through a process of angiogenesis in part driven by VEGFA ${ }^{37}$. In the Opn4 null mouse, retinal angiogenesis is promiscuous and the vascular networks show elevated density and ectopic sprouts ${ }^{13}$. For this reason we also assessed development of the retinal vasculature in the Gna11', Gnaq ${ }^{f l}, \mathrm{Opn}^{\text {cre }}$ allelic series. Counts of branch-points revealed a gene dosage-dependent elevation in vascular density (Fig. 2E-G) but, as with the hyaloid vessel phenotype, no apparent contribution from GNA11. When we compared the phenotype severity for GNAQ and OPN4 loss-of-function (as proportion of control, white number Figs. $2 \mathrm{G}, \mathrm{H}$ ), as with the hyaloid vessel phenotype, we found that GNAQ can fully account for the activity of OPN4. Combined, the hyaloid vessel and retinal angiogenesis phenotypes of mice with GNAQ loss-of-function in Opn4-expressing cells suggest that OPN4 signaling for this vascular development pathway is coupled through GNAQ.

\section{Activation of GNAQ at fetal stages reverses the vascular consequences of Opn4 loss-of- function.}

To further assess the relationship between light responses, OPN4, GNAQ and vascular development, we determined whether we could reverse the vascular consequences of darkrearing or Opn4 mutation by activating GNAQ signaling only in Opn4-expressing cells. We designed these experiments so that we could activate GNAQ only during late gestation and in fetal mice, not in the pregnant dam. This addressed the question of whether OPN4-dependent signaling responses occurred in fetal mice.

For these experiments, we employed a ROSA26-based allele expressing hM3Dq ${ }^{38}$, a DREADD (Designer Receptors Exclusively Activated by Designer Drugs) based on a G-coupled receptor that activates GNAQ in response to the small molecule ligand $\mathrm{CNO}^{39}$. By activating $R 26$ $h M 3 D q$ with Opn4 ${ }^{\text {cre }}$ (Fig. 3A), we could obtain expression in Opn4-expressing cells and by injecting CNO into the dam in late gestation (Fig. 3B), we could restrict GNAQ activation to fetal stages. We also performed crosses that excluded the possibility of GNAQ activation in the dam. We used this experimental strategy to generate a series of control and experimental mice that were assessed for both hyaloid vessel regression and retinal vascular density.

For experiments to determine whether GNAQ activation could reverse the vascular consequences of Opn4 loss-of-function, we crossed an Opn4 ${ }^{\text {cre/cre; }}$ R26-hM3Dq male with an $O p n 4^{+/-}$female. This provided appropriate control and experimental genotypes in fetal mice but 
excluded the possibility that the dam could respond to CNO. We then injected CNO daily from E16 to day of birth (P1) and harvested retina and hyaloid at P8 for quantification of vascular structures. As expected, Opn4 $4^{\text {cre/- }}$ pups showed hyaloid persistence at P8 (Fig. 3C, and the dark gray bar in G). By contrast, Opn4 ${ }^{\text {cre/-; }}$ R26-hM3Dq pups injected with CNO showed a normal number of hyaloid vessels (Fig. 3D, and the dark blue bar in G). Similarly, Opn4 ${ }^{\text {cre/- }}$ animals showed an abnormally elevated retinal vascular density at P8 (Fig. 3F, H) and this was reversed when Opn4 ${ }^{\text {cre/- }}$ was combined with R26-hM3Dq and gestational CNO (Fig. 3F, H). These data show that for the vascular development pathways in the eye, GNAQ activity can substitute for OPN4 within ipRGCs. The experiment also makes a strong case that this vascular development pathway is dependent on the activity of melanopsin at fetal stages.

\section{Activation of GNAQ in ipRGCs at fetal stages reverses the vascular consequences of dark rearing.}

As a second step in this analysis, we determined whether fetal stage GNAQ activation could reverse the vascular consequences of dark-rearing (DD, Fig. 3B). For this analysis, we crossed a male Opn $4^{\text {cre/cre }}$ homozygote with a female $R 26-h M 3 D q$ heterozygote. This meant that half the fetal mice had expression activation of $\mathrm{hM} 3 \mathrm{Dq}$ in melanopsin-expressing neurons, but importantly, the dam did not carry Opn $4^{\text {cre }}$ and therefore could not activate expression of R26$h M 3 D q$. Thus, any response to the CNO ligand could only be attributed to fetal Opn4-expressing cells. When we performed the analysis, we found that CNO injected into pregnant female mice from E15 through E19 reversed the vascular consequences of dark rearing (Fig. 4A-H). As expected, DD conditions resulted in significantly elevated hyaloid vessel numbers at P8 (Fig. 4A, $C, D)$. In the absence of late gestational injections of CNO the elevated number of hyaloid vessels was not significantly changed with or without hM3Dq (Fig. 4C, D). However, when CNO was injected from E15 to E19 into DD, Opn4 $4^{\text {cre }}$ R26-hM3Dq mice, the normally elevated number of hyaloid vessels remained at the level observed in the LD control (compare Fig. 4E, blue bar with $4 \mathrm{C}$, both bars). When we quantified retinal vascular branch-points (Fig. 4F, G, H), we observed a very similar pattern where Opn $4^{\text {cre }}, R 26-h M 3 D q$, and gestational CNO were all required to reverse the retinal vascular overgrowth of DD to control levels (Fig. 4H). These data indicate that activation of GNAQ signaling in Opn4-expressing cells can substitute for light in late gestation and give normal regulation of vascular development. 


\section{Discussion}

Here we have combined experimental strategies to address the question of whether melanopsin activation during fetal stages can regulate vascular development in the mouse eye. Within the retina, OPN4 expression is restricted to a subset of retinal ganglion cells (RGCs) that are, in adult mice, intrinsically photosensitive ${ }^{9,14,40}$. Opn4 is expressed as early as E10.5 $5^{34}$ and OPN4 protein is detected in the retina around $\mathrm{E} 15^{41}$ in mouse embryos. Like other opsins, melanopsin (aka Opsin 4, OPN4) is a G-coupled receptor ${ }^{29,42}$ and functions predominantly via Gnaq/11 coupling ${ }^{24,27,28}$. Few studies have suggested alternate G-protein coupling for melanopsin in both in vivo ${ }^{26}$ and in expression systems ${ }^{29-31}$. Current data on melanopsin G-protein coupling is from adult or neonatal mice. We focused our efforts on late gestational ages and our data indicates that melanopsin in the fetal retina uses conventional Gnaq/11-coupling for ocular vascular development.

In the first step of this analysis, we determined whether the loss of GNAQ function in Opn4expressing cells produced the same phenotype as a melanopsin (OPN4) loss-of-function. We show mice with the Opn4 ${ }^{\text {cre }}$; Gnaq $q^{f / f l}$ genotype had the retinal vascular overgrowth and hyaloid vessel persistence characteristic of the Opn4 null and of dark rearing from late gestation ${ }^{13}$. Furthermore, the addition of germ-line Gna11 homozygosity to Gnaq conditional deletion from Opn4-expressing cells did not exacerbate vascular phenotypes. These data argue that GNAQ, and not GNA11, mediates melanopsin-dependent vascular development. As a final step in this series of experiments we determined whether GNAQ gain of function was sufficient to reverse the vascular phenotypes that result from dark rearing or from Opn4 mutation. We tested whether GNAQ activation in Opn4-expressing cells only during the fetal stages of mouse development was sufficient to reverse the vascular changes that normally result from dark rearing or Opn4 lossof-function. Our findings showed that when GNAQ gain-of-function was restricted to Opn4expressing cells in late mouse gestation, this was sufficient to overcome the absence of light or the absence of OPN4 for the vascular development pathway.

These data thus make a strong case that if the vascular development of a mouse eye is to be normal, GNAQ must be activated in Opn4-expressing cells from E16 to E19 of gestation. The ability to revert the Opn4 loss-of-function phenotype with conditional activation of GNAQ also suggests that the activity of OPN4 and GNAQ in Opn4-expressing cells are directly related. The ability to revert the vascular consequences of dark rearing also indicate that the fetal retina normally responds to environmental light. We show that the fetal light response pathway regulating vascular development requires GNAQ but not GNA11 (Fig. 2). Involvement of GNA14 
in the fetal ipRGC response remains a possibility though this is unlikely given the quantitative correspondence between Opn4 and Gnaq loss-of-function.

Multiple members of the Gnaq/11 subfamily, including Gna14, Gnaq and Gna11, can participate in melanopsin phototransduction in vivo ${ }^{24,27,28}$. However, a recent study showed that Opn4 ${ }^{\text {cre }}$; Gna11\%; $G n a q^{\text {fl/fl }}$ mice like those used here, many of the photic responses ascribed to melanopsin remain normal ${ }^{26}$. The present analysis, showing that GNA11 does not contribute to vascular development responses, is consistent with the documented lack of a multielectrode array response in the neonatal retina of Gna11; Gna14 null mice ${ }^{26}$. However, the Gnaq loss-of-function phenotype, coupled to the rescue of the OPN4 loss-of-function phenotype by GNAQ gain-offunction, makes a compelling case that GNAQ is an important signaling mediator of OPN4 activity in fetal stages and is consistent with the observation of GNAQ presence in all M1-5 ipRGCs in adult mice ${ }^{24}$. One possible explanation for these apparently discrepant findings is that different functions of melanopsin are mediated by distinct types of signaling response and are likely to be age-specific. One scenario is that perhaps the vascular development pathway assessed here relies on the activity of GNAQ while the pupillary reflex and circadian photoentrainment rely on other G-proteins that are expressed at a later stage of ipRGC development.

The data presented here make a strong case that the eye of a fetal mouse uses light as normal developmental cue. Though we currently do not understand the biological rationale with precision, it is quite likely that the fetal eye light response prepares the eye for visual function. Certainly, vascular development of the eye must be closely regulated for normal vision but it is also likely that other responses to fetal OPN4 stimulation will be identified. OPN4 is highly conserved within the vertebrates both in primary sequence and function. This raises the fascinating possibility that the human fetal eye is also light responsive in a way that regulates vascular development. This idea has received support from a recent clinical study that associates first trimester fetal light exposure with severe forms of retinopathy of prematurity, an ocular blood vessel overgrowth of the premature infant ${ }^{43}$.

\section{Acknowledgements}

We are indebted to Dr. Ute Hochgeschwender for providing R26-LSL-Gq-DREADD mice prior to publication. We are also grateful to Dr. Ute Hochgeschwender and Dr. Shawnta Y. Chaney for critiquing this MS. We thank Mr. Paul Speeg for excellent technical assistance. We acknowledge funding from the National Eye Institute (EY021636-01 to RAL and DRC) in support of this work. 


\section{Materials and Methods}

\section{Animals}

All animal experimentation was carried out using protocols approved by the Institutional Animal Care and Use Committee at Cincinnati Children's Hospital Medical Center. Mouse lines used in this study have been previously described: Opn4 $4^{\text {cre }}\left(\mathrm{ref}^{44}\right)$ Opn4- $\left(\mathrm{ref}^{10}\right)$, Ai14 (JAX \#007914), Gnaq ${ }^{f l}\left(\mathrm{ref}^{45}\right)$, Gna11 $\left(\mathrm{ref}^{45}\right)$ and R26-LSL-Gq-DREADD ( $\left.\mathrm{ref}^{38}\right)$. Genotyping information is described in the cited publications. Mice were housed in a 12:12 hour light-dark (LD) cycle. For dark-rearing experiments, pregnant dam was moved to constant darkness (DD) after lights OFF at E15. Clozapine-N-oxide (CNO, Sigma \#CO832-5MG) stock solution was prepared at $10 \mathrm{mg} / \mathrm{ml}$ in $100 \%$ DMSO and injected into the dam at $1 \mathrm{mg} / \mathrm{kg}$ body weight to activate Gq-DREADD $(h M 3 D q)$. Dam was injected daily at lights ON in LD cycle or at subjective lights ON for animals in DD from embryonic day E16 to day of birth. For DD animals, injections were performed under dim red light. Littermate controls were used for all experiments to accommodate for differences in mouse strains.

\section{Hyaloid and retinal labeling}

Hyaloid vessel and retinal flat-mount preparations have been previously described (Rao 2013). Hyaloid preparations and retinae were labeled with Hoechst 33258 and isolectin (1:500, Thermo Fisher Scientific \#121411), respectively. Hyaloid vessel quantification has been described previously (Rao 2013). Retinal vessel density was quantified by counting vessel junctions using ImageJ for at least ten $320 \mu \mathrm{m}^{2}$ fields per retina. We used paired T-test to assess statistical significance. In the G-protein immunohistochemical study, retinae for cryosections were fixed at room temperature for 2 hours in 4\% PFA and then cryopreserved in 30\% sucrose and sectioned at $10 \mu \mathrm{m}$ followed by antigen retrieval with ice cold sodium citrate buffer for 10 minutes. Sections and flat-mounts were incubated in anti-GNAQ/GNA11 (1:100, Santa Cruz sc-392) at $4^{\circ} \mathrm{C}$ for $1-3$ days followed by secondary antibody (1:500, Thermo Fisher Scientific \#A-21206) overnight at $4^{\circ} \mathrm{C}$.

\section{References}

1. Palczewski, K. et al. Crystal Structure of Rhodopsin: A G Protein-Coupled Receptor. Sci. (New York, NY) 289, 739-745 (2000).

2. Palczewski, K. Chemistry and biology of vision. Journal of Biological Chemistry 287, 1612-1619 (2012).

3. Palczewski, K. \& Saari, J. C. Activation and inactivation steps in the visual transduction 
pathway. Current Opinion in Neurobiology 7, 500-504 (1997).

4. Nathans, J. In the eye of the beholder: visual pigments and inherited variation in human vision. Cell 78, 357-60 (1994).

5. Provencio, I., Jiang, G., De Grip, W. J., Hayes, W. P. \& Rollag, M. D. Melanopsin: An opsin in melanophores, brain, and eye. Proc. Natl. Acad. Sci. 95, 340-345 (1998).

6. Davies, W. I. L. et al. An extended family of novel vertebrate photopigments is widely expressed and displays a diversity of function. Genome Res. 25, 1666-1679 (2015).

7. Lucas, R. J. et al. Measuring and using light in the melanopsin age. Trends in Neurosciences 37, 1-9 (2014).

8. Kumbalasiri, T. \& Provencio, I. Melanopsin and other novel mammalian opsins. Experimental Eye Research 81, 368-375 (2005).

9. Panda, S. et al. Melanopsin is required for non-image-forming photic responses in blind mice. Science 301, 525-527 (2003).

10. Panda, S. et al. Melanopsin (Opn4) requirement for normal light-induced circadian phase shifting. Science 298, 2213-6 (2002).

11. Lucas, R. J. et al. Diminished pupillary light reflex at high irradiances in melanopsinknockout mice. Science 299, 245-247 (2003).

12. Johnson, J. et al. Melanopsin-dependent light avoidance in neonatal mice. Proc. Natl. Acad. Sci. U. S. A. 107, 17374-17378 (2010).

13. Rao, S. et al. A direct and melanopsin-dependent fetal light response regulates mouse eye development. Nature 494, 243-246 (2013).

14. Hattar, S., Liao, H. W., Takao, M., Berson, D. M. \& Yau, K. W. Melanopsin-containing retinal ganglion cells: architecture, projections, and intrinsic photosensitivity. Science $\mathbf{2 9 5}$, 1065-70 (2002).

15. Provencio, I., Rollag, M. D. \& Castrucci, A. M. Photoreceptive net in the mammalian retina. This mesh of cells may explain how some blind mice can still tell day from night. Nature 415, 493 (2002).

16. Bergström, A. L., Hannibal, J., Hindersson, P. \& Fahrenkrug, J. Light-induced phase shift in the Syrian hamster (Mesocricetus auratus) is attenuated by the PACAP receptor antagonist PACAP6-38 or PACAP immunoneutralization. Eur. J. Neurosci. 18, 25522562 (2003).

17. Morin, L. P., Blanchard, J. H. \& Provencio, I. Retinal ganglion cell projections to the hamster suprachiasmatic nucleus, intergeniculate leaflet, and visual midbrain: Bifurcation and melanopsin immunoreactivity. J. Comp. Neurol. 465, 401-416 (2003).

18. Sollars, P. J. et al. Melanopsin and non-melanopsin expressing retinal ganglion cells innervate the hypothalamic suprachiasmatic nucleus. Vis. Neurosci. 20, 601-610 (2003).

19. Hannibal, J. \& Fahrenkrug, J. Target areas innervated by PACAP-immunoreactive retinal ganglion cells. Cell Tissue Res. 316, 99-113 (2004).

20. Hattar, S. et al. Central projections of melanopsin-expressing retinal ganglion cells in the mouse. J. Comp. Neurol. 497, 326-349 (2006).

21. Baver, S. B., Pickard, G. E., Sollars, P. J. \& Pickard, G. E. Two types of melanopsin retinal ganglion cell differentially innervate the hypothalamic suprachiasmatic nucleus and the olivary pretectal nucleus. Eur. J. Neurosci. 27, 1763-1770 (2008).

22. Chen, S.-K., Badea, T. C. \& Hattar, S. Photoentrainment and pupillary light reflex are mediated by distinct populations of ipRGCs. Nature 476, 92-5 (2011).

23. Sekaran, S. et al. Melanopsin-dependent photoreception provides earliest light detection in the mammalian retina. Curr. Biol. 15, 1099-1107 (2005).

24. Hughes, S. et al. Using siRNA to define functional interactions between melanopsin and multiple G Protein partners. Cell. Mol. Life Sci. 72, 165-179 (2014).

25. Graham, D. M. et al. Melanopsin ganglion cells use a membrane-associated rhabdomeric phototransduction cascade. J Neurophysiol 99, 2522-2532 (2008). 
26. Chew, K. S., Schmidt, T. M., Rupp, A. C., Kofuji, P. \& Trimarchi, J. M. Loss of Gq/11 genes does not abolish melanopsin phototransduction. PLoS One 9, (2014).

27. Panda, S. et al. Illumination of the melanopsin signaling pathway. Science 307, 600-604 (2005).

28. Qiu, X. et al. Induction of photosensitivity by heterologous expression of melanopsin. Nature 433, 745-749 (2005).

29. Bailes, H. J. \& Lucas, R. J. Human melanopsin forms a pigment maximally sensitive to blue light $(\lambda \max \approx 479 \mathrm{~nm}$ ) supporting activation of $\mathrm{G}(\mathrm{q} / 11)$ and $\mathrm{G}(\mathrm{i} / \mathrm{o})$ signalling cascades. Proc. Biol. Sci. 280, 20122987 (2013).

30. Newman, L. A., Walker, M. T., Brown, R. L., Cronin, T. W. \& Robinson, P. R. Melanopsin Forms a Functional Short-Wavelength Photopigment. Biochemistry 42, 12734-12738 (2003).

31. Kankanamge, D., Ratnayake, K., Samaradivakara, S. \& Karunarathne, A. Melanopsin (Opn4) employs Gai and Gßy as major signal transducers. J. Cell Sci. (2018). doi:10.1242/jcs.212910

32. Tu, D. C. et al. Physiologic diversity and development of intrinsically photosensitive retinal ganglion cells. Neuron 48, 987-999 (2005).

33. Wong, K. Y. A retinal ganglion cell that can signal irradiance continuously for 10 hours. J. Neurosci. 32, 11478-11485 (2012).

34. Tarttelin, E. E. et al. Expression of opsin genes early in ocular development of humans and mice. Exp. Eye Res. 76, 393-396 (2003).

35. Ito, M. \& Yoshioka, M. Regression of the hyaloid vessels and pupillary membrane of the mouse. Anat. Embryol. (Berl). 200, 403-411 (1999).

36. Lobov, I. B. et al. WNT7b mediates macrophage-induced programmed cell death in patterning of the vasculature. Nature 437, 417-421 (2005).

37. Gerhardt, H. et al. VEGF guides angiogenic sprouting utilizing endothelial tip cell filopodia. J. Cell Biol. 161, 1163-1177 (2003).

38. Zhu, H. et al. Cre-dependent DREADD (Designer Receptors Exclusively Activated by Designer Drugs) mice. Genesis 54, 439-46 (2016).

39. Urban, D. J. \& Roth, B. L. DREADDs (Designer Receptors Exclusively Activated by Designer Drugs): Chemogenetic Tools with Therapeutic Utility. Annu. Rev. Pharmacol. Toxicol. 55, 399-417 (2015).

40. Ruby, N. F. et al. Role of melanopsin in circadian responses to light. Science 298, 22112213 (2002).

41. McNeill, D. S. et al. Development of melanopsin-based irradiance detecting circuitry. Neural Dev. 6, 8 (2011).

42. Melyan, Z., Tarttelin, E. E., Bellingham, J., Lucas, R. J. \& Hankins, M. W. Addition of human melanopsin renders mammalian cells photoresponsive. Nature 433, 741-745 (2005).

43. Yang, M. B., Rao, S., Copenhagen, D. R. \& Lang, R. A. Length of day during early gestation as a predictor of risk for severe retinopathy of prematurity. Ophthalmology 120, 2706-2713 (2013).

44. Ecker, J. L. et al. Melanopsin-expressing retinal ganglion-cell photoreceptors: Cellular diversity and role in pattern vision. Neuron 67, 49-60 (2010).

45. Wettschureck, N. et al. Absence of pressure overload induced myocardial hypertrophy after conditional inactivation of Galphaq/Galpha11 in cardiomyocytes. Nat. Med. 7, 12361240 (2001). 


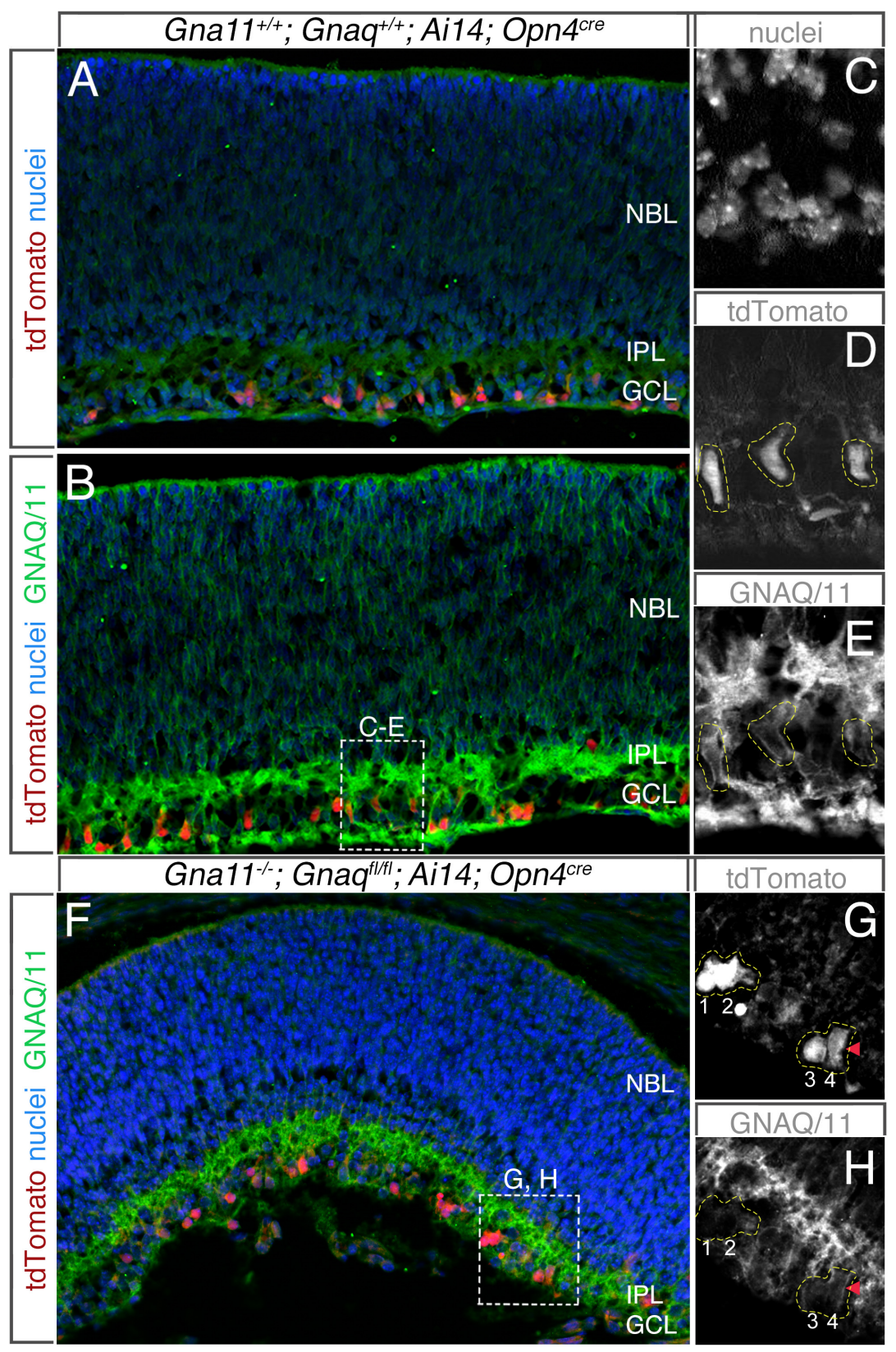

Figure 1. GNAQ loss-of-function in ipRGCs can be generated with Opn4 ${ }^{\text {cre }}$.

(A-E) Cryosections from P2, Gna111+-; $\mathrm{Gnaq}^{+/+}$; Ai14; Opn44 ${ }^{\text {cre }}$ mice were labeled for nuclei (A, B, blue, C, grayscale), tdTomato (A, B, red, D, grayscale) and GNAQ/11 (B, green, E, grayscale, A no primary antibody control). C-E represent different channels from region demarcated in (B). In ( $E$, yellow dashed lines), GNAQ/11 labeling is associated with the membrane of three ipRGCs according to the presence of tdTomato as shown in (D, yellow dashed lines). (F-H) Cryosections from E18, Gna11 Opn4 $4^{\text {cre }}$ mice were labeled for nuclei $(F$, blue), tdTomato $(F$, red, $G$, grayscale) or for GNAQ ( $F$, green, $H$, grayscale). G-H represent different channels from region demarcated in $(F)$. In $(H)$, the cells labeled 1-4 are ipRGCs according to the expression of tdTomato (G). Unlike ipRGCs in the control sections (D, E), cells 1 and 2 (G, H, yellow dashed line) do not show GNAQ/11 membrane labeling. Cells 3 and 4 (G, H, red arrowhead) show some GNAQ labeling and may therefore have escaped cre-mediated deletion of both $\mathrm{Gnaq}^{f l}$ alleles. NBL neuroblast layer, IPL inner plexiform layer, GC ganglion layer 

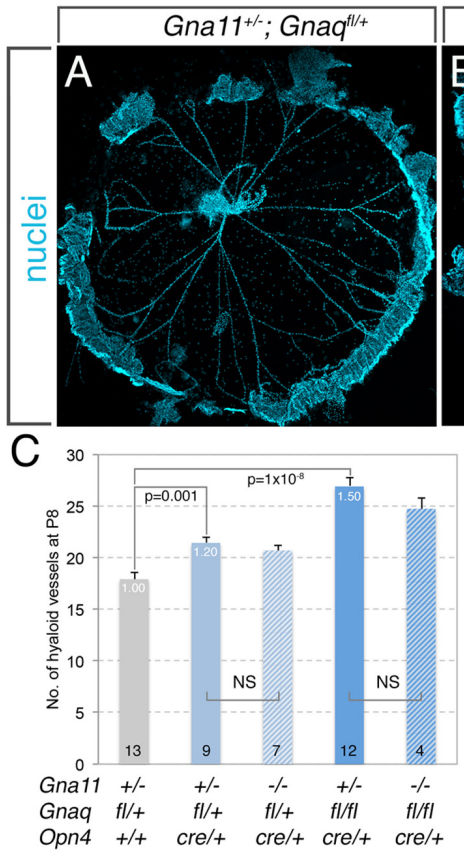
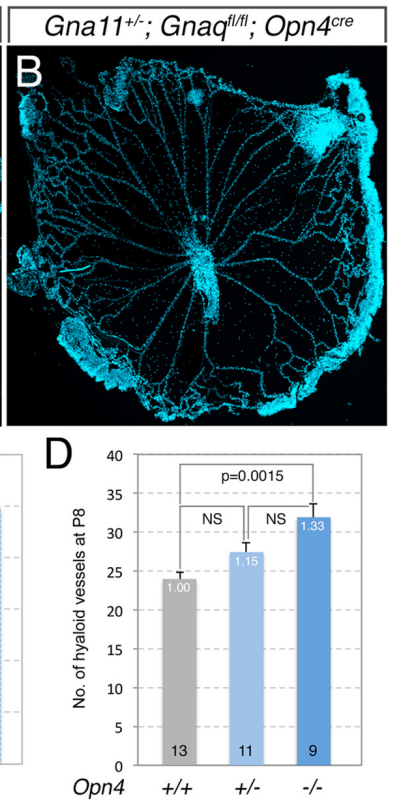

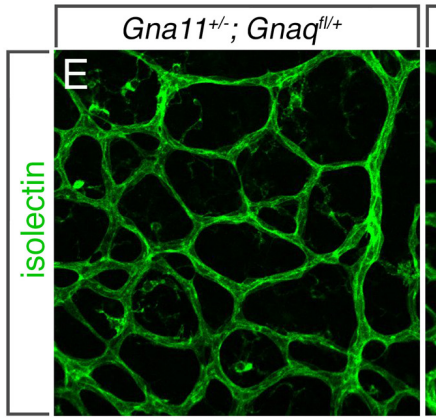

G

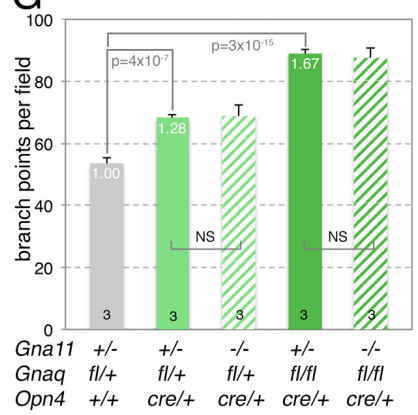

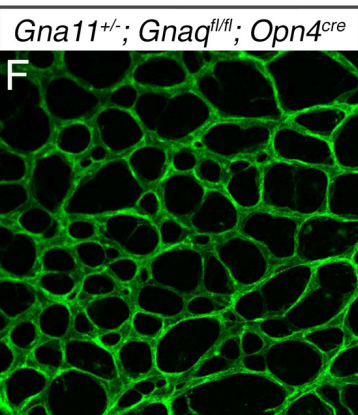

$\mathrm{H}$

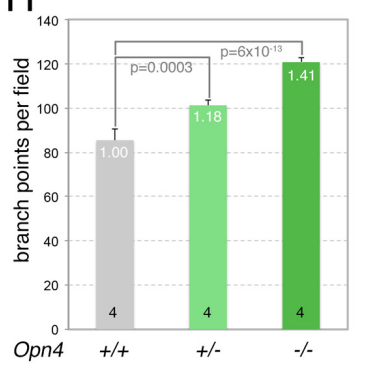

Figure 2. Gnaq loss-of-function in ipRGCs results in hyaloid vessel persistence and retinal vascular overgrowth. (A, B) Hoechst 33258-labeled hyaloid vessel preparations for control Gna11+/; $\mathrm{Gnaq}^{f / /+}$ (A) and experimental Gna11+/; Gnaq $q^{f / f t /}$; Opn4 ${ }^{\text {cre }}$ (B) mice at P8. (C) Number of hyaloid vessels in P8 mice of the indicated genotypes. (D) For comparison with (C), number of hyaloid vessels in P8 Opn4 wild type, heterozygote and homozygote mice. (E, F) Flat mount retina preparations

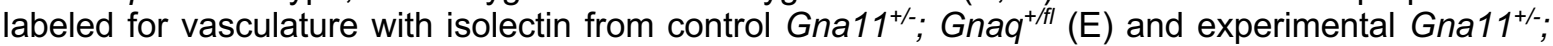
Gnaqf/ff; Opn4 ${ }^{\text {cre }}(\mathrm{F})$ mice at P8. (G) Number of retinal vascular branch-points in P8 mice of the indicated genotypes. $(\mathrm{H})$ Number of retinal vascular branch-points in P8 mice in Opn4 wild type, heterozygote and homozygote mice. Data represented as mean \pm S.E.M. Sample size is shown at the base of the each histogram and represents number of animals from multiple litters. $p$ values calculated by Student's T-test. $p$ values as labeled, NS, not significant, for $(C, D, G, H)$ white number at top of bars indicates the proportion of the control value for each panel. 


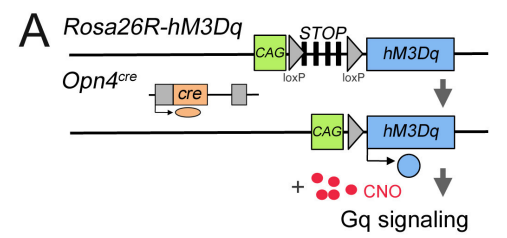

B CNO at lights ON (ZTO or CTO)
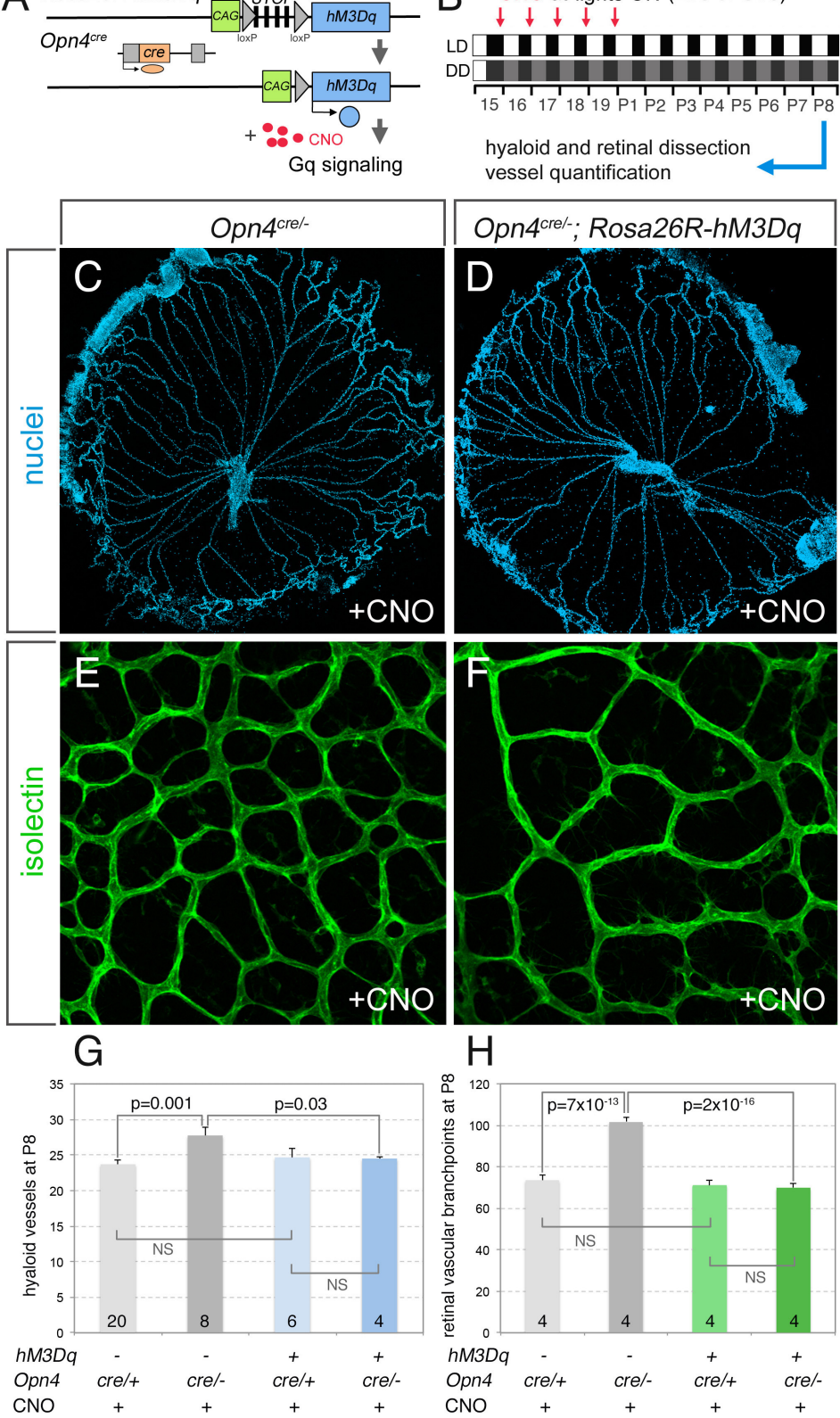

Figure 3. GNAQ gain-of-function in ipRGCs during gestation reverses the postnatal vascular consequences of Opn4 mutation. (A) Schematic describing how the R26-hM3Dq and Opn4cre alleles can be combined with the artificial hM3Dq ligand CNO to give GNAQ signaling only in ipRGCs. (B) Experimental design for chemogenetic approach using hM3Dq/CNO. CNO ligand is injected into dam from E16 to P1 (red arrow) either in LD lighting cycle or after the transition to DD at E16 at lights ON (ZTO for LD or subjective lights ON, CTO for DD). At P8, hyaloid and retinal vessels are quantified. Hyaloid $(C, D)$ and retinal $(E, F)$ vessel preparations from Opn4 $4^{\text {cre/ }}(\mathrm{C}, \mathrm{E})$ and Opn4 $4^{\text {cre/; }}, h M 3 D q(\mathrm{D}, \mathrm{F})$ mice that have received CNO from E16 to E19. (G) Quantification of P8 hyaloid vessel numbers in mice of the labeled genotypes and CNO exposure. $(\mathrm{H})$ Quantification of P8 retinal vascular branch-points in mice of the labeled genotypes and CNO exposure. LD, normal lighting conditions, DD, constant darkness after E15. cre, cre recombinase coding region, CAG, chicken $\beta$ actin promoter. $p$ values as labeled; NS, not significant. Data represented as mean \pm SEM. Sample size is shown at the base of each histogram and represents number of animals from three independent experiments. $p$ values calculated using Student's T-test. 


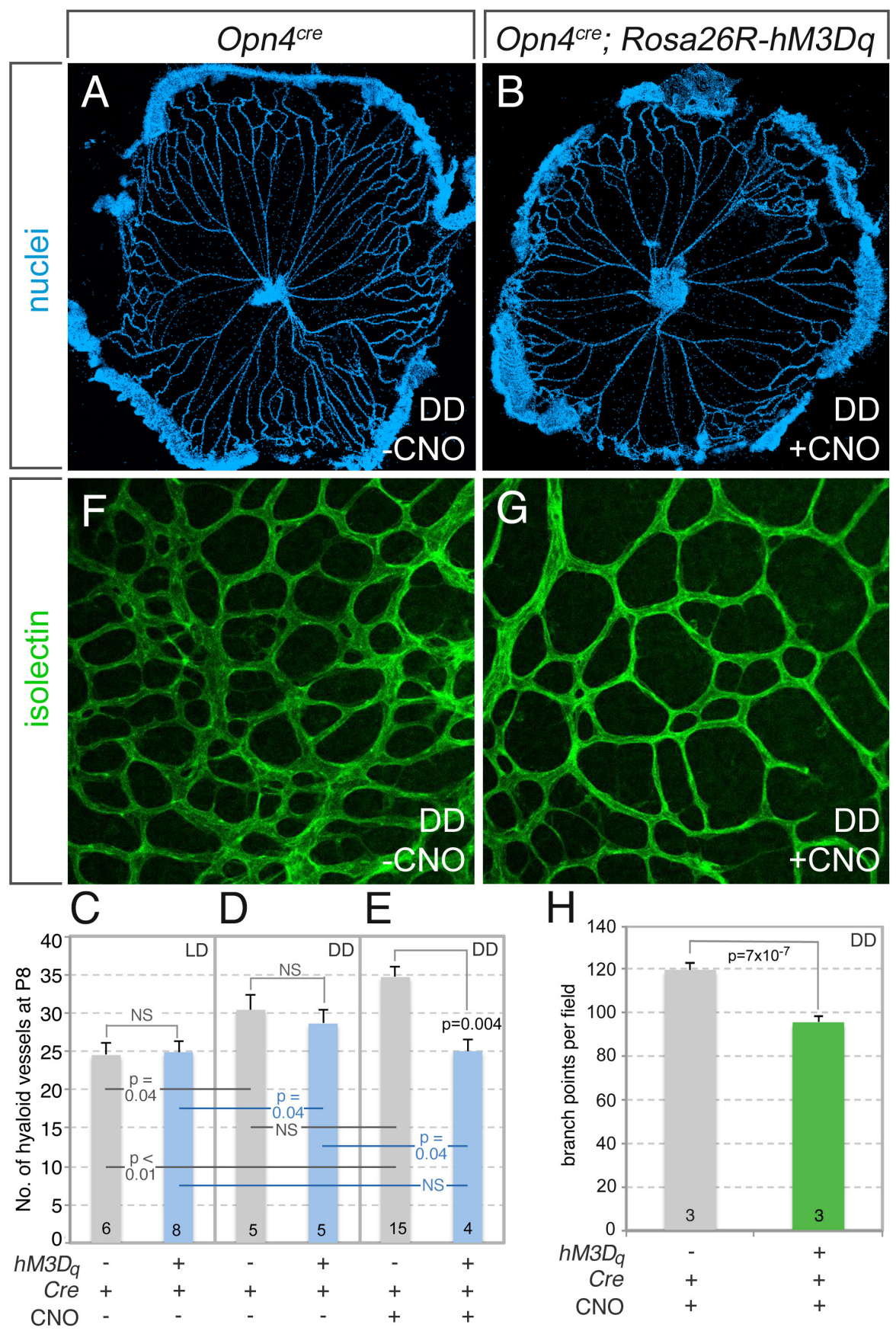

Figure 4. GNAQ gain-of-function in ipRGCs during gestation reverses the postnatal vascular consequences of dark-rearing. (A, B) Hyaloid vessel preparations at P8 for Opn $4^{\text {cre }}(\mathrm{A})$ and $\mathrm{Opn} 4^{\mathrm{cre}}$; $h M 3 D q(\mathrm{~B})$ mice where dam was moved to DD at E15 and injected with CNO from E16 to day of birth P1 (see Fig. 3b for experimental design). (C-E) Quantification of P8 hyaloid vessel numbers in mice of the labeled genotypes and CNO exposure. $(F, G)$ Retinal vessel preparations at P8 for Opn4 ${ }^{\text {cre }}(\mathrm{F})$ and Opn4 ${ }^{\text {cre }}$; $h M 3 D q(\mathrm{G})$ mice moved to DD as above. $(\mathrm{H})$ Quantification of P8 retinal vascular branch-points in mice of the labeled genotypes and CNO exposure. LD, normal lighting conditions, DD, constant darkness after E15. $p$ values as labeled; NS, not significant. Data represented as mean \pm S.E.M. Sample size is shown at the base of each histogram bar and represents number of animals from three independent experiments. $p$ values calculated using Student's T-test. 